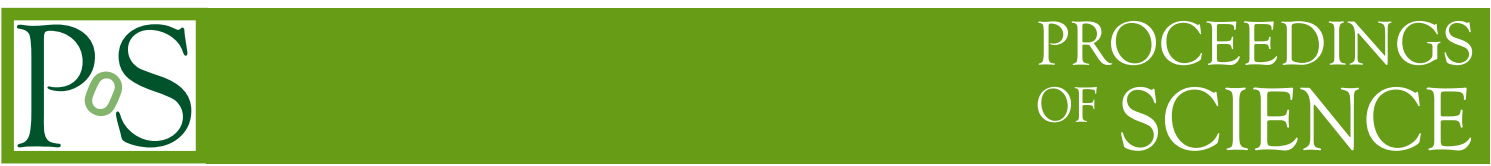

\title{
CKM Fits
}

\section{Valentin Niess*, on behalf of the CKMfitter group}

LPC, Clermont (UMR6533), Université Blaise Pascal, CNRS/IN2P3, Aubière, France;

E-mail: niess@in2p3.fr

\begin{abstract}
We present the results of a global fit of the CKM matrix parameters to experimental data within the Standard Model theoretical context. We follow a frequentist approach to handle statistical uncertainties and use a conservative Rfit scheme to deal with ill-modeled theoretical systematics, mostly arising from strong interaction effects. The current experimental data are found to be consistent with the Standard-Model framework establishing the KM mechanism as the dominant source of $\mathrm{CP}$ violation at the electroweak scale. Yet, measurements exhibit an ongoing tension between the branching ratio of $B^{+} \rightarrow \tau^{+} v$ purely leptonic decays and $\sin (2 \beta)$ derived from charmonium $B$ decays. We further discuss this tension in the light of two particular new physics scenarii. Namely, we discuss extensions of the Standard Model to two Higgs doublet with Type II couplings, or by assuming additional new physics short distance contributions to $B$ 's in $\Delta F=2$ transitions, by following a model-independent approach.
\end{abstract}

Flavor Physics and CP Violation 2009

May 27-June 12009

Lake Placid, NY, USA

* Speaker. 


\section{Introduction}

In the Standard Model (SM), the weak charged-current transitions mix quarks of different generations. The mixing amplitudes are described by the 3 by 3 unitary Cabibbo-Kobayashi-Maskawa (CKM) matrix [1]. For three generations of quarks, the CKM matrix is completely defined by only 4 real parameters, whose choice in not unique. One of these parameters is a non-vanishing phase which is currently the only established source of CP violation in the SM. The lepton sector could also exhibit similar sources of CP violation. For our analysis we use a unitary-exact Wolfensteinlike parameterisation [2, 3] of the CKM matrix by letting:

$$
\lambda=\frac{\left|V_{u d}\right|}{\sqrt{\left|V_{u d}^{2}\right|+\left|V_{u s}^{2}\right|}}, A \lambda^{2}=\frac{\left|V_{c b}\right|}{\sqrt{\left|V_{u d}^{2}\right|+\left|V_{u s}^{2}\right|}}, \bar{\rho}+i \bar{\eta}=-\frac{V_{u d} V_{u b}^{*}}{V_{c d} V_{c b}^{*}},
$$

where $(\bar{\rho}, \bar{\eta})$ defines the apex of the $B_{d}$ unitarity triangle (UT). Note that these 4 CKM parameters are derived from the moduli of CKM matrix elements or from a 4 product invariant [3], hence there are rephasing invariant. Additionally, the CKM matrix elements can be expanded in powers of the Cabibbo angle $\lambda=\sin \left(\theta_{C}\right)$, while preserving the matrix unitarity up to any order of the series.

The expansion parameter $\lambda$ is accurately determined, at $0.3 \%$ level, from superallowed nuclear transitions, yielding $\left|V_{u d}\right|$, and from semileptonic kaon decays, yielding $\left|V_{u s}\right|$. The parameter $A$, determined with an accuracy of $3 \%$, requires the knowledge of $\left|V_{c b}\right|$ measured from charmed $B$ semileptonic decays. The apex $(\bar{\rho}, \bar{\eta})$ of the UT is less accurately known. It is derived from sides and angle measurements of the UT. One of the challenges is that the precise extraction of observables related to these electroweak parameters is complicated by the presence of strong interaction effects.

\section{The Global CKM Fit}

We perform a global fit of the CKM parameters values in order to best match the observed experimental data, $\vec{x}_{o b s}$, to their SM theoretical predictions. With this aim we distinguish two categories of uncertainties: statistical ones, which are assumed to be Gaussian distributed with known standard deviation and correlations, and theoretical systematics, for which no statistical distribution can be safely assumed. Hence, the latter are considered as additional fit parameters bounded to a confidence interval. This dedicated treatment of theoretical systematics is the so called RFit scheme [3].

\subsection{General Fit Procedure}

The core fit procedure and the interpretation of the fits results rely on frequentist hypothesis testing tools. In the following we give a practical description of our fit procedure. For justifications we refer the reader to reference $[\rrbracket$. We start by splitting our parameter vector $\vec{\theta}$ in two distinct sets. On one side are the scanned parameters of interest, for example $(\bar{\rho}, \bar{\eta})$. Let this component be $\vec{\mu}$. The remaining parameters are considered as nuisance parameters and the corresponding component is noted $\vec{v}$. For a given value $\vec{\mu}_{0}$ of the component $\vec{\mu}$, which is our test null hypothesis 
expressed as the following assumption, $H_{0}: \vec{\mu}=\vec{\mu}_{0}$, we can compute the observed value taken by the likelihood function, $\mathscr{L}\left(\vec{\mu}, \vec{v} ; \vec{x}_{o b s}\right)=\mathrm{p}\left(\vec{x}=\vec{x}_{o b s} ; \vec{\mu}, \vec{v}\right)$. The latter likelihood is the objective function to optimise for our fit, such that 'best match' should be understood as: maximising the likelihood. Then, for the hypothesis test statistic we consider the maximum likelihood ratio, or relative likelihood, defined as:

$$
\lambda(\vec{x} ; \vec{\mu})=\frac{\sup _{\vec{v}} \mathscr{L}(\vec{\mu}, \vec{v} ; \vec{x})}{\sup _{\vec{\mu}, \vec{v}} \mathscr{L}(\vec{\mu}, \vec{v} ; \vec{x})},
$$

which in our case is more conveniently expressed by considering the log likelihood, setting $\Delta \chi^{2}=$ $-2 \ln (\lambda)$. Note however that despite the notation, the $\Delta \chi^{2}$ values are not necessarily $\chi^{2}$ distributed. Technically, the $\Delta \chi^{2}$ value obtained from equation (2.1) is the variation of the likelihood fit residuals by fixing the scanned parameters or using their best match, letting them free in the fit.

In order to interpret the observed test value $\Delta \chi_{o b s}^{2}$, at $\vec{x}=\vec{x}_{o b s}$, we quote the corresponding statistical significance, or the critical p-value, of the data under the null hypothesis $H_{0}: \vec{\mu}=\vec{\mu}_{0}$, as:

$$
\text { p-value }\left(\vec{\mu}_{0}, \vec{v} ; \vec{x}_{o b s}\right)=\mathrm{P}\left(\Delta \chi^{2} \geq \Delta \chi_{o b s}^{2} ; \vec{\mu}_{0}, \vec{v}\right)=\int_{\Sigma_{o b s}} \mathrm{p}\left(\vec{x} ; \vec{\mu}_{0}, \vec{v}\right) d \vec{x}
$$

with $\Sigma_{o b s}=\left\{\vec{x} ; \Delta \chi^{2}\left(\vec{x} ; \vec{\mu}_{0}\right) \geq \Delta \chi_{o b s}^{2}\right\}$. It must be pointed out that generally speaking the statistical distribution of the $\Delta \chi^{2}$ can be non trivial and in particular it can be strongly affected by the true value of the nuisance parameters $\vec{v}$, through the sampling of $\vec{x}$. Hence, quoting a statistical significance for the solely scanned parameter values can be ambiguous. However, under regularity conditions defined by Wilks theorem, in the asymptotic regime $\Delta \chi^{2}$ is to be $\chi^{2}$ distributed, independently of $\vec{v}$. The latter asymptotic regime is assumed for most results presented in this paper, involving a large number of observables and nuisance parameters. If not, we rely on dedicated toy Monte-Carlo studies to sample the $\Delta \chi^{2}$ distribution more accurately.

Finally, we can repeat the fit procedure as a scan for various values of the parameter set of interest $\vec{\mu}$. By construction, a confidence region of level CL for the scanned parameter is provided by the set of parameter values for which the corresponding p-value exceeds $1-\mathrm{CL}$, as p-value $\left(\vec{\mu} ; \vec{x}_{o b s}\right) \geq 1-\mathrm{CL}$. When the asymptotic regime is assumed for the $\Delta \chi^{2}$ distribution, the confidence intervals we obtain consist in profile likelihood intervals, or so called MINOS intervals [円].

\subsection{Illustrated RFit Scheme}

To illustrate the problem, let us consider a simplified example, fully solvable by analytical means. Let us have a single observable, $x$, whose theoretical prediction is simply given as $x=\mu$. We further assume that the observation of $x$ is smeared by a centred Gaussian distributed error of standard deviation $\sigma$, and biased by a systematic $\Delta_{x}$, which is to lie within $[-\Delta ; \Delta]$. Hence, $x$ is Gaussian distributed of central value $\mu+\Delta_{x}$ and standard deviation $\sigma$. Following equation (2.1), for a given value of $\mu$ one checks that the test statistic, $\Delta \chi^{2}$, writes as: 


$$
\Delta \chi^{2}(x ; \mu)= \begin{cases}\frac{(|x-\mu|-\Delta)^{2}}{\sigma^{2}}, & \text { if }|x-\mu|>\Delta \\ 0, & \text { elsewhere }\end{cases}
$$

Note that the previous expression extends to a more general scheme where the $\chi^{2}$ depends on multiple observables with Gaussian distributed statistic errors. In the latter, provided the systematic $\Delta_{x}$ is not correlated among the observables, the minimum of the $\chi^{2}$ as respect to $\Delta_{x}$ is simply obtained by substituting the component $\left(x-\mu-\Delta_{x}\right)^{2} / \sigma^{2}$ in the $\chi^{2}$ by $\Delta \chi^{2}(x ; \mu)$ as given by equation (2.3).

Back to our simplified example, the p-value is computed from equation (2.2), recalling that $x$ is Gaussian distributed. It yields:

$$
\text { p-value }\left(\mu, \Delta_{x} ; x_{o b s}\right)=\left\{\begin{array}{ll}
\frac{1}{2}\left(\operatorname{erfc}\left(\frac{\left|x_{o b s}-\mu\right|-\Delta_{x}}{\sqrt{2} \sigma}\right)+\operatorname{erfc}\left(\frac{\left|x_{o b s}-\mu\right|+\Delta_{x}}{\sqrt{2} \sigma}\right)\right), & \text { if }\left|x_{o b s}-\mu\right|>\Delta \\
1, & \text { elsewhere }
\end{array} .\right.
$$

Note that in the limit $\Delta \rightarrow 0$, one recovers the classical p-value for a Gaussian distributed error. From the latter equation (2.4) it is also seen that even though the test statistic $\Delta \chi^{2}$ does not depend on the nuisance parameter $\Delta_{x}$, its statistical distribution and so its p-value does. A conservative method to escape the latter issue is to consider the worst case over all values of the nuisance parameter, the so called supremum p-value [5]. In the simplified example we consider the supremum p-value is always achieved at boundaries for $\Delta_{x}= \pm \Delta$.

On figure 1 we compare the result of the Rfit scheme to what one would get by assuming that the systematic $\Delta_{x}$ is statistically distributed, uniformly over the range $[-\Delta ; \Delta]$. Supremum p-value is considered. One sees that the RFit approach is always more conservative. In particular as one is dominated by systematics, $\Delta / \sigma \rightarrow \infty$, see the lower right plot,the $R f i t$-value becomes rectangular, centred on the observation and of span $\pm \Delta$, resulting in a flat significance over the systematics range. But, if assuming a uniform distribution, one gets a triangular p-value, favouring the observation over other values within the systematics range.

\subsection{Observables for the Global Fit}

In table 1 we summarised the various categories of observable we use as input to the global CKM fit as well as the related experimental sources and theory ingredients. More details and references are given in [3, 6]. Among all these observables, only two have been modified since our last summer 2008 update: the branching ratio (BR) of the $B^{+} \rightarrow \rho^{+} \rho^{0}$ channel updated by the BaBar collaboration [7] and HFAG [8] average of $\sin (2 \beta)$ from charmonium $B$ decays.

Because the weak effects we are interested in are entangled with strong interaction effects confining the quarks, hadronic inputs are also required. These hadronic inputs severely contribute to the fit accuracy and to CKM parameter uncertainties. In particular, they limit the precision on the determination of the observables involving processes with loops such as, $\Delta m_{d}, \Delta m_{s},\left|\varepsilon_{K}\right|$ and also the tree decay $B^{+} \rightarrow \tau^{+} v$. On the contrary, hadronic contributions to the semileptonic $K_{\ell 3}$ decays 

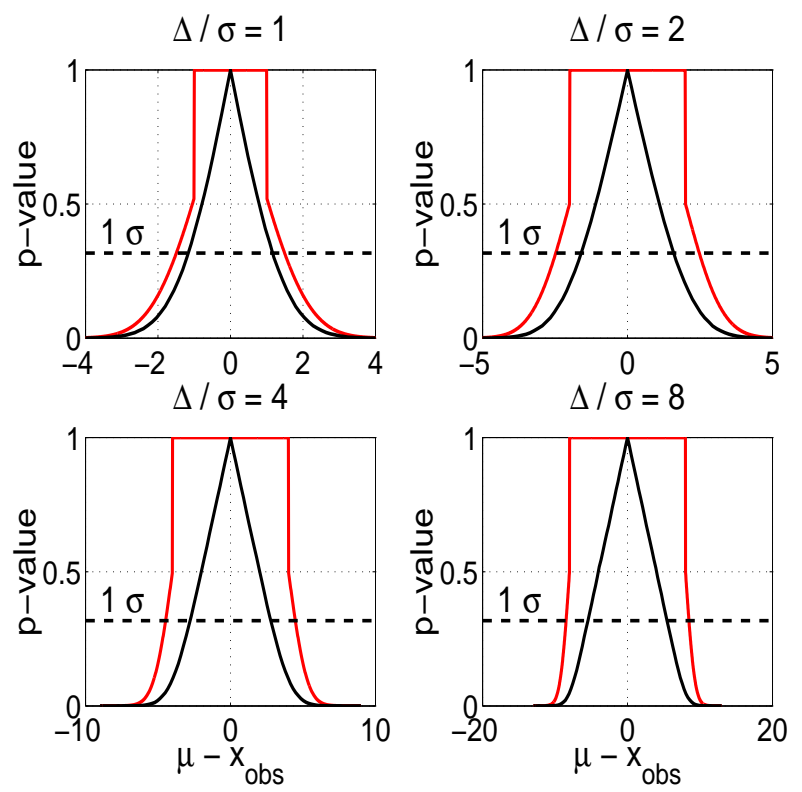

Figure 1: Illustrated RFit scheme for various values of the ratio $\Delta / \sigma$ of the systematic to the statistic error. The red curve stands for the supremum RFit p-value while the black curve is the result for a statistic treatment of the systematic, uniformly distributed over the range $\pm \Delta$. The horizontal dotted line indicates the equivalent $1 \sigma$ confidence level for a Gaussian distributed error, given as $1-\mathrm{CL}=\operatorname{erfc}(1 / \sqrt{2}) \simeq 0.3173$.

are surprisingly under excellent control. For our fit, we mostly rely on lattice QCD (LQCD) simulations to estimate these hadronic inputs. Accuracies of lattice predictions have steadily improved, with precisions of a few percent achieved for example on the ratio of $B_{s}$ to $B_{d}$ decay constants, $f_{B_{s}} / f_{B_{d}}$. However, it is required to combine the results from different collaborations with various statistic and systematic uncertainties. This game is particularly tricky since LQCD predictions are now dominated by systematics. In the absence of a general averaging consensus, we have set up our own averaging of these results following a reproducible Educated RFit scheme [11].

\subsection{Global Fit Results}

Combining the various observables summarised in table 1 lour global fit best guess shows good agreement with experimental data. The p-value for the SM hypothesis is of $45 \%$ (equivalent to $0.8 \sigma$

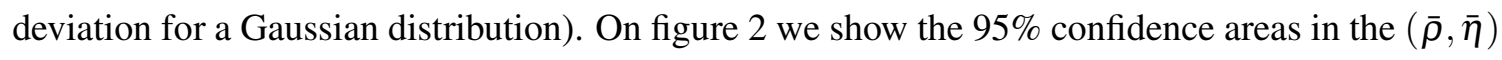
plan for individual constraints, as well as for their combination. One sees that the fit is dominated by $\sin (2 \beta), \Delta m_{d} / \Delta m_{s}$ and $\alpha$ observables. While only two of these observables would be enough to determine the apex of the UT, these 3 observables over-constrain it by exhibiting an impressive agreement. Hence, we can confidently say that the $\mathrm{KM}$ mechanism is at work for $\mathrm{CP}$ violation and dominant in $B$ decays.

From individual scans we find the following values for the CKM parameters, quoting $1 \sigma$ confidence intervals: $\lambda=0.22521 \pm 0.00082, A=0.8116_{-0.0241}^{+0.0097}, \bar{\rho}=0.139_{-0.027}^{+0.025}, \bar{\eta}=0.341_{-0.015}^{+0.016}$. Additional numerical results can be found in reference [ [6]. 


\begin{tabular}{|c|c|c|}
\hline Observable Category & Experimental Sources & Theory Methods \& Inputs \\
\hline$\left|V_{u d}\right|$ & superallowed $\beta$-decays & Towner \& Hardy, PRC77 025501 (2008) \\
\hline$\left|V_{u s}\right|$ & $K_{\ell 3}$, FlaviaNet [9] average & $f_{+}^{K \pi}(0)=0.964(5)(\mathrm{RBC}-\mathrm{UKQCD})$ \\
\hline$\left|V_{c b}\right|$ & $B \rightarrow X_{c} \ell v($ HFAG excl.+incl.) & $40.59(38)(58) \cdot 10^{-3}, \mathrm{FF}$ and/or OPE \\
\hline$\left|V_{u b}\right|_{\mathrm{SL}}$ & $B \rightarrow X_{u} \ell v$ (HFAG excl.+incl.) & $(\dagger) 3.87(9)(46) \cdot 10^{-3}, \mathrm{FF}$ and/or OPE \\
\hline $\mathscr{B}\left(B^{+} \rightarrow \tau^{+} \boldsymbol{v}\right)\left(\Rightarrow\left|V_{u b}\right|\right)$ & 2008 average: BaBar and Belle & $(\dagger) f_{B_{s}} / f_{B_{d}}=1.196(8)(23), f_{B_{s}}=228(3)(17)$ \\
\hline$\Delta m_{d}\left(\Rightarrow\left|V_{t d}\right|\right)$ & HFAG average $B_{d}^{0}-\bar{B}_{d}^{0}$ mixing & $(\dagger) \hat{B}_{B_{s}} / \hat{B}_{B_{d}}=1.05(2)(5), f_{B_{s}} / f_{B_{d}}$ \\
\hline$\Delta m_{s}\left(\Rightarrow\left|V_{t s}\right|\right)$ & $\mathrm{CDF} B_{s}^{0}-\bar{B}_{s}^{0}$ mixing & $(\dagger) \hat{B}_{B_{s}}=1.23(3)(5), f_{B_{s}}$ \\
\hline$\left|\varepsilon_{K}\right|$ & $\bar{K}^{0}-\bar{K}^{0}$ PDG 2008 average & $\Delta S=2$ amplitudes, $\hat{B}_{K}=0.721(5)(40)$ \\
\hline$\alpha / \phi_{2}$ & $B \rightarrow \pi \pi, \rho \rho, \rho \pi$ B fact. average & Isospin SU(2), Gronau \& London [10] \\
\hline$\beta / \phi_{1}$ & Charmonium B decays, HFAG & $\sin \left(2 \beta_{c c}\right)=0.671(23)$ \\
\hline$\gamma / \phi_{3}$ & $B^{-} \rightarrow D^{(*)} K^{(*)-}$, B fact. average & GLW, ADS, GGSZ \\
\hline
\end{tabular}

Table 1: Various relevant observables used as input to the global CKM fit. Numerical values that have been computed using the Educated RFit averaging scheme are tagged with a dagger as ( $\dagger$ ). The upper part of the table corresponds to $\mathrm{CP}$ conserving observables while the lower part is $\mathrm{CP}$ violating quantities. Full information on fit inputs can be found in reference [6].

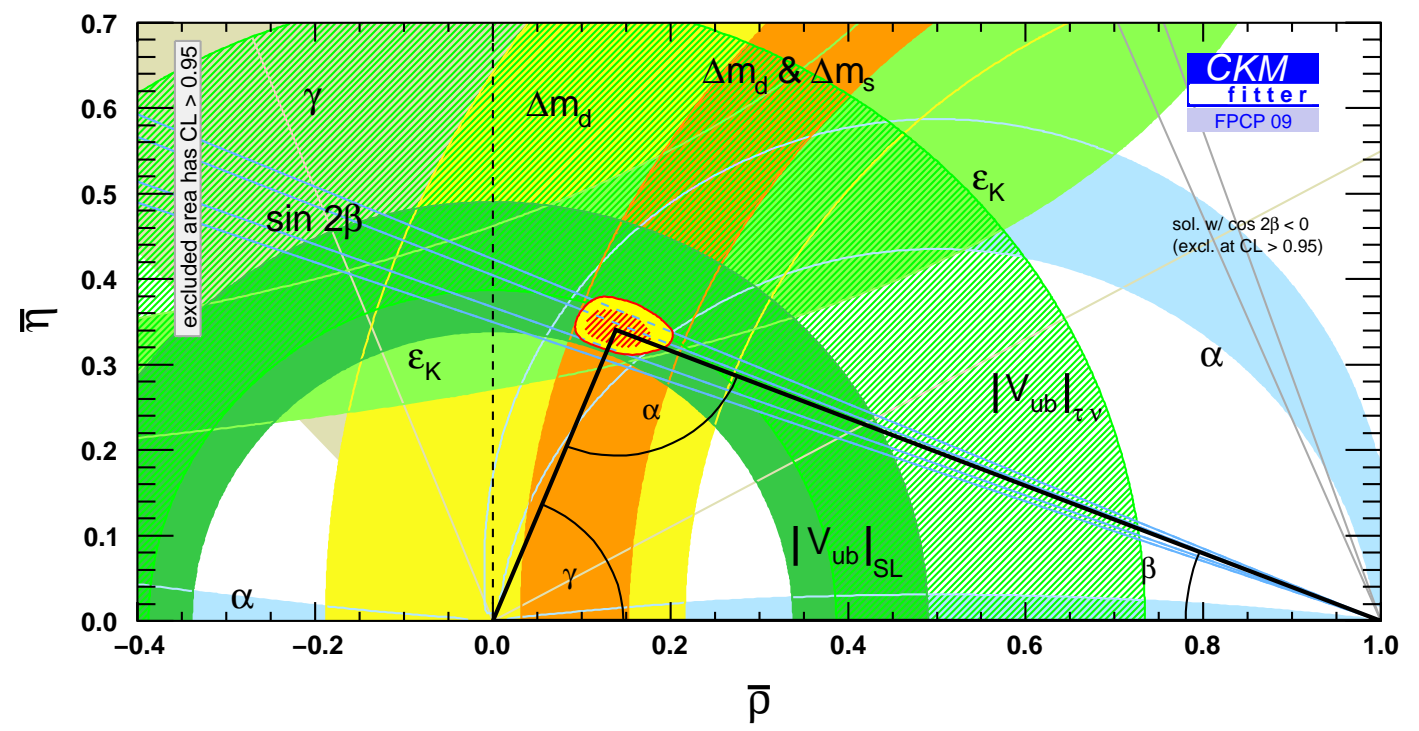

Figure 2: $95 \% \mathrm{CL}$ individual and global constraints in the $(\bar{\rho}, \bar{\eta})$ plan from the global CKM fit. The red hashed region of the global combination corresponds to $68 \% \mathrm{CL}$. 


\subsection{Update of the $\alpha$ Angle}

The angle $\alpha$ is extracted from $B \rightarrow \pi \pi, \rho \rho$ and $\rho \pi$ decays using rates and asymmetries. Because the direct transition is polluted by penguin contributions with similar CKM amplitudes, but different weak phases, one does not measure directly the angle $\alpha$, as it is the case for the angle $\beta$. Rather one measures an effective angle $\alpha_{e f f}$. Further assuming SU(2) isospin symmetry one can however extract $\alpha$ from a global fit to $B \rightarrow h h$ rates and asymmetries [3, 10]. Comparing the three different cases $(\pi \pi, \rho \rho$ and $\rho \pi)$, one finds that the smallest penguin pollution, and thus the most stringent bound, comes from $\rho \rho$ modes.

Following BaBar update of the BR of the $B^{+} \rightarrow \rho^{+} \rho^{0}$ mode the angle $\alpha$ is now determined with a good accuracy, at the level of $5 \%$ or less: $\alpha=\left(89.0_{-4.2}^{+4.4}\right)^{\circ}$, reaching similar accuracy than the angle $\beta$. Compared to Summer 08 results, $\alpha=\left(88.2_{-4.8}^{+6.1}\right)^{\circ}$, the improved global accuracy comes from a factor of 2 improvement in the extraction of $\alpha$ from $\rho \rho$ modes. Technically, this improvement by a factor of 2 results from two previously degenerated mirror solutions, due to discrete ambiguities inherent to the method, that collapsed into a single one. Though it might seem somewhat incidental it was checked from toy Monte-Carlo studies [11] that the current situation is quite likely since penguins contributions are small.

Additionally, to study possible systematics from the $\mathrm{SU}(2)$ symmetry assumption, we have also modeled isospin breaking effects [11]. It was checked that even sizable isospin breaking contributions have only a very mild impact on the determination of the angle $\alpha$ at 95\% CL.

\section{Tensions and New Physics Studies}

Looking at individual observables, the only significant deviation we do see as regard to our fit best guess result concerns the value of the $B^{+} \rightarrow \tau^{+} v$ BR. For the later we got a $2.4 \sigma$ deviation of the measurement to our fit.

Treating hadronic uncertainties within the Educated RFit scheme we dot not see a significant difference between $\left|V_{u b}\right|$ determined inclusively from semi leptonic $B \rightarrow X_{u} \ell v$ decays, $\left|V_{u b}\right|_{\text {incl. }}=$ 4.38(16)(57) $\cdot 10^{-3}$, and from exclusive ones, $\left|V_{u b}\right|_{\text {excl. }}=3.46(11)(46) \cdot 10^{-3}$ [B], 11]. Neither do we see a deviation between the measurement and the indirect prediction of $\left|\varepsilon_{K}\right|$ from CKM parameters. Note that the latter theoretical prediction suffers from $\sim 7 \%$ uncertainties from $\left|V_{c b}\right|$ which plays in to the $4^{\text {th }}$ power and $5 \%$ from hadronic uncertainties on the value of the bag parameter, $\hat{B}_{K}$.

\section{1 $B^{+} \rightarrow \tau^{+} v$ versus $\sin (2 \beta)$ Tension}

The deviation of $B^{+} \rightarrow \tau^{+} v$ BR can be mostly resolved if we drop $\sin (2 \beta)$ from the fit. The point is that the combination of $B^{+} \rightarrow \tau^{+} v$ and $\sin (2 \beta)$ defines two solutions for the UT apex that are incompatible with other observables: $\Delta m_{d} / \Delta m_{s}$ and $\alpha$. However, when discarding either $B^{+} \rightarrow$ $\tau^{+} v$ or $\sin (2 \beta)$ one can perfectly fit the remaining observables. Hence there is an inconsistency between $B^{+} \rightarrow \tau^{+} v$ (too high) and $\sin (2 \beta)$ (too low) experimental values or with our theoretical interpretation of these observables, badly-estimated hadronic parameters for example.

The $B^{+} \rightarrow \tau^{+} v$ experimental $\mathrm{BR}$ is obtained from a weighted mean of BaBar and Belle measurements using two different tags [12]. For the experimental combination we have: $\mathscr{B}\left[B^{+} \rightarrow\right.$ $\left.\tau^{+} v\right]=(1.73 \pm 0.35) \cdot 10^{-4}$, while our indirect theoretical prediction from CKM parameters is 

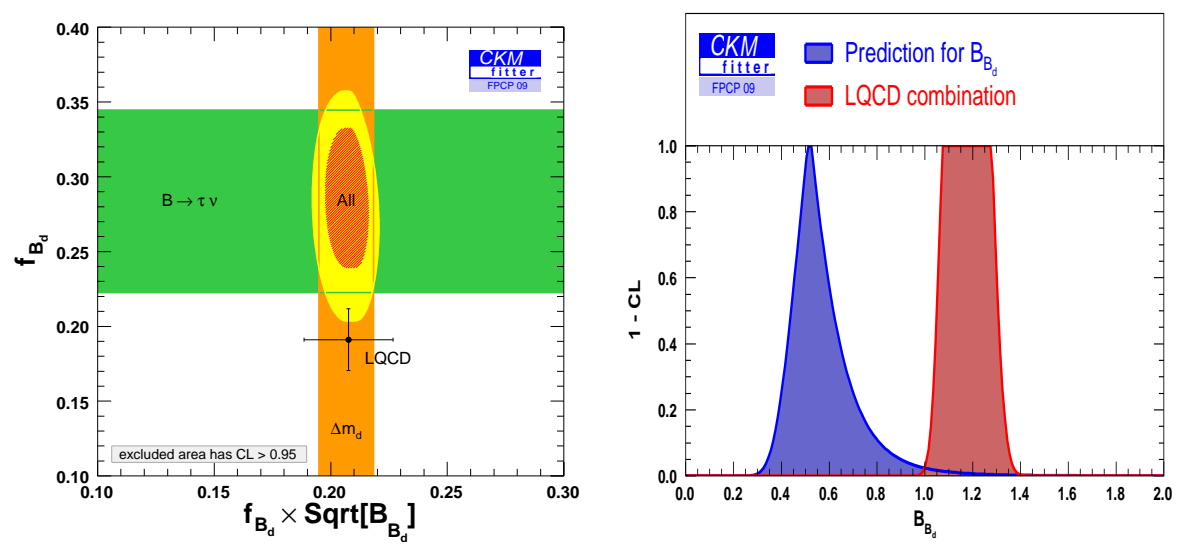

Figure 3: Measurements of hadronic parameters, $f_{B_{d}}, f_{B_{d}} \sqrt{\hat{B}_{B_{d}}}$ and $\hat{B}_{B_{d}}$. On the left figure, colored areas show the $95 \%$ confidence area for the corresponding parameters. The Green constrain is derived from CKM observables, not including $\Delta m_{d}$ while the orange one is derived not using $\mathscr{B}\left[B^{+} \rightarrow \tau^{+} v\right]$. The yellow area is the combined constrain and the dashed red zone is the corresponding $1 \sigma$ area. The superimposed black cross indicates our prediction from LQCD dominated by systematic uncertainties, with $1 \sigma$ error bars. On the right figure, the blue curve is the p-value function for $\hat{B}_{B_{d}}$, not including LQCD inputs, while the red one is our prediction from solely LQCD data. The two predictions disagree at $2.4 \sigma$. We recall that the confidence intervals of level CL are given by values of $\hat{B}_{B_{d}}$ for which the p-value is greater than $1-\mathrm{CL}$. At $95 \% \mathrm{CL}$ it yields $\hat{B}_{B_{d}} \in[1.01 ; 1.36]$ from LQCD inputs, to be compared to $\hat{B}_{B_{d}} \in[0.34 ; 0.90]$ from CKM observables only, not including LQCD inputs.

only $\left(0.796_{-0.093}^{+0.154}\right) \cdot 10^{-4}$. The four measurements used for the experimental average are consistent and in agreement with the hypothesis of a Gaussian distributed uncertainty, with a p-value of $11 \%$ $(1.6 \sigma)$ according to a two sided $\chi^{2}$ test. For $\sin (2 \beta)$ experimental value we recall that we use HFAG [8] average of charmonium $B$ decays. The corresponding set of measurements also looks consistent.

$\mathscr{B}\left[B^{+} \rightarrow \tau^{+} v\right]$ theoretical prediction suffers from hadronic uncertainties on the value of the decay constant, $f_{B_{d}}$. We extract the later from our own lattice averages of $f_{B_{s}}$ and $f_{B_{s}} / f_{B_{d}}$, yielding $f_{B_{d}}=191.1 \pm 20.7 \mathrm{MeV}$, in perfect agreement with recent LQCD estimates [13]. Reversing the problem, we can estimate hadronic inputs: $f_{B_{d}}, f_{B_{d}} \sqrt{\hat{B}_{B_{d}}}$ and $\hat{B}_{B_{d}}$ [12] from our sole CKM observables, not making use of LQCD predictions. The estimates we obtain, within the SM, are shown on figure 3. One can see that while the LQCD prediction for the product $f_{B_{d}} \sqrt{\hat{B}_{B_{d}}}$ is consistent with our estimate, $f_{B_{d}}$ or $\hat{B}_{B_{d}}$ individually disagree at more than $2 \sigma$. Therefore, only a correlated change of the two latter parameters, that is increasing $f_{B_{d}}$ and decreasing $\hat{B}_{B_{d}}$, while keeping the product $f_{B_{d}} \sqrt{\hat{B}_{B_{d}}}$ mostly unchanged would allow to accomodate the current experimental value of $\mathscr{B}\left[B^{+} \rightarrow \tau^{+} v\right]$ into the global CKM fit.

\subsection{Charged-Higgs Contributions in 2HDM(II)}

Going one step farther, let us assume that both experimental measurements of $B^{+} \rightarrow \tau^{+} v$ and $\sin (2 \beta)$ and hadronic estimates of $f_{B_{d}}$ and $\hat{B}_{B_{d}}$ are close to their true expectation, and see how we 
could accommodate all these in a New Physics (NP) scenario. There are two possible effects of NP models. Either the model increases the prediction for $\mathscr{B}\left[B^{+} \rightarrow \tau^{+} v\right]$ or it decreases the prediction for $\sin (2 \beta)$ from charmonium $B$ decays.

Considering the first scenario, $B^{+} \rightarrow \tau^{+} v$ transitions mediated by a charged-Higgs, $H^{+}$, instead of a $W^{+}$boson, could significantly increase the BR within a fine-tuned scenario of the charged-Higgs mass to the $B$ meson mass. Charged-Higgs would arise in extensions of the SM to Two Higgs Doublet Models (2HDM). Of particular interest are type II models which mimic the flavour structure of the SM as well as the Supersymmetric (SUSY) Higgs sector at tree level. However, from a global analysis, also considering $K$ and $D$ mesons leptonic and semileptonic decays this fine-tuned charged-Higgs scenario is disfavored at more than 95\% CL [14], assuming type II 2HDM. Further considering observables the like $B \rightarrow X_{s} \gamma$, most sensitive to charged-Higgs contributions through top quark loop couplings, the $2 \mathrm{HDM}$ (II) scenario is constrained to a decoupling solution, with an indirect limit on the charged-Higgs mass of $m_{H^{+}} \geq 304 \mathrm{GeV}$ at $95 \%$ CL [11]].

\subsection{New Physics in B Mixing}

The expectation for $\sin (2 \beta)$ from charmonium $B$ decays could be decreased by NP contributions to the meson mixing. Let us consider the scenario where NP only affects the short distance part of the $\Delta B=2$ transitions, the like previously discussed in [11, 12, 15]. Using a modelindependent parameterisation, it is found that the tension between $\mathscr{B}\left[B^{+} \rightarrow \tau^{+} v\right]$ and $\sin (2 \beta)$ can be fully resolved if interpreting it as as additional NP phase in $B$ mixing, of $\Phi_{d}^{N P}=-\left(12_{-9}^{+6}\right)^{\circ}$ at 95\% CL. Though this is no striking evidence for an additional NP correction in the $B$ 's sector, we point out that whatever $\mathscr{B}\left[B^{+} \rightarrow \tau^{+} v\right]$ is taken into account or not, sizable NP contributions to the $B_{d}$ mixing are still allowed: up to $\sim-18^{\circ}$ on the phase and $\sim 50 \%$ on the amplitude, at 95\% CL.

Additionally, in the $B_{s}$ system the measurement of the angle $\phi_{s}=2 \beta_{s}$, not included in the standard global CKM fit, is also a source of tension. The angle $\phi_{s}$ is measured at Tevatron by CDF and D0 [8], in $B_{s} \rightarrow J / \Psi \phi$ channel. It deviates at $2.2 \sigma$ from the SM expectation. Combining the 2.4 deviation in the $B_{d}$ system, arising from the $B^{+} \rightarrow \tau^{+} v$ versus $\sin (2 \beta)$ tension, with the tension in the $B_{s}$ system, arising from $\phi_{s}$, the SM hypothesis is disliked at $2.5 \sigma$ in a generic scenario of NP in both $B_{d}$ and $B_{s}$ mixing.

\section{Conclusion}

Following developments on both experimental ( $B$ factories, Tevatron) and theoretical (LQCD) sides, it is seen that the SM accurately fits most of the current experimental data. This establishes the KM mechanism as the dominant source of $\mathrm{CP}$ violation at the electroweak scale. The only significant deviation that we observe consists in an ongoing tension between $\mathscr{B}\left[B^{+} \rightarrow \tau^{+} v\right]$ and $\sin (2 \beta)$ derived from charmonium $B$ decays. The significance of this tension is $2.4 \sigma$ within the SM. It could be fully absorbed by NP contributions to the $B_{d}$ mixing. Conversely, sizable NP contributions to both $B_{d}$ and $B_{s}$ mixing are still allowed.

Improvements on $B$ related LQCD predictions as well as more precise experimental measurements (LHC, super $B$ factories) are expected in the future. These data will allow to confirm or infirm the interpretation of the combined $2.5 \sigma$ tension in the $B$ mixing as additional NP contributions. Furthermore, the accurate direct measurement of the angle gamma $\left(\sim 5^{\circ}\right.$ at $\mathrm{LHCb}$ with 
$2 \mathrm{fb}^{-1}$ [16], which is only one year of regular data taking, and $\sim 2^{\circ}$ at superB factories with $75 \mathrm{ab}^{-1}[17]$ ) will provide an additional consistency test of the KM mechanism.

\section{Acknowledgments}

For help and support with this work, I thank my colleagues from the CKMfitter group. I also thank the FPCP 09 organisers and actors for an interesting and fruitful conference, though somewhat snowy for end of may in Lake Placid, NY.

\section{References}

[1] N. Cabibbo, Phys. Rev. Lett. 10, 531 (1963).

M. Kobayashi and T. Maskawa, Prog. Theor. Phys. 49, 652 (1973).

[2] L. Wolfenstein, Phys. Rev. Lett. 51, 1945 (1983).

[3] J. Charles et al. [CKMfitter Group], Eur. Phys. J. C 41, 1 (2005) [arXiv:hep-ph/0406184];

[4] F. James, Statistical Methods in Experimental Physics, $2^{\text {nd }}$ edition, World Scientific (2006).

[5] J. Heinrich, L. Lyons, Annu. Rev. Part. Sci. 57, 145 (2007).

[6] Moriond 09 update at URL: http://ckmfitter.in2p3.fr/plots_Moriond09/.

[7] The BaBar Collaboration (B. Aubert et al.), Phys. Rev. Lett. 102, 141802 (2009).

[8] The Heavy Flavour Averaging Group (HFAG), arXiv:0808.1297 [hep-ex]; http://www.slac.stanford.edu/xorg/hfag/ and references therein.

[9] FlaviaNet Working Group on kaon decays, arXiv:0801.1817 [hep-ph] (2008).

[10] M. Gronau and D. London, Phys. Rev. Lett. 65, 3381 (1990).

[11] V. Tisserand, Moriond 09 EW, arXiv:0905.1572 [hep-ph] (2009).

[12] O. Deschamps, arXiv:0810.3139 [hep-ph] to appear in the proceedings of ICHEP 08, Philadelphia (2008).

[13] C. Davies, these proceedings (slide 14).

Fermilab Lattice and MILC Collaborations (C. Bernard et al.), arXiv:0904.1895 [hep-lat] (2009).

[14] O. Deschamps et al. [CKMfitter Group], arXiv:0907.5135 [hep-ph] (2009).

[15] A. Lenz and U. Nierste, JHEP 0706:072 (2007).

[16] O. Deschamps [LHCb Collaboration], these proceedings.

[17] M. Bona et al. [SuperB Collaboration], arXiv:0709.0451 [hep-ex]. 